DOI https://doi.org/10.18551/rjoas.2021-01.20

\title{
EMPHYSEMATOUS CARBUNCLE IN CATTLE
}

\author{
Kapustin A.V. ${ }^{1,2}$, Laishevtsev A.I. ${ }^{1,2 *}$, Motorygin A.V. ${ }^{3}$ \\ ${ }^{1}$ Federal Scientific Center - All-Russian Research Institute of Experimental Veterinary \\ Medicine named after K.I. Skryabin and Y.R. Kovalenko of the Russian Academy \\ of Sciences, Moscow, Russia \\ ${ }^{2}$ Laboratory of Biological Control and Antimicrobial Resistance, Orel State University \\ named after I.S. Turgenev, Orel City, Russia \\ ${ }^{3}$ Russian State Center for Animal Feed and Drug Standardization and Quality, \\ Moscow, Russia \\ *E-mail: cvat19@mail.ru
}

\begin{abstract}
Emphysematous carbuncle is an acute infectious disease of cattle, which occurs enzootically in disadvantageous areas during the pasture and, less often, the stall period. Cases of emphysematous carbuncle in sheep have also been described, but unlike cases in cattle, the disease is often provoked by the ingress of the pathogen through wounds, which is why emphysematous carbuncle in sheep should be considered as a disease associated with malignant edema. The pathogen of the emphysematous carbuncle, Clostridium chauvoei, causes extensive damage to various systems and tissues of the body, accompanied by rapidly growing toxicosis, severe focal muscle damage in the form of crepitant necrosis, serous-hemorrhagic infiltration and damage to adjacent subcutaneous tissue, which leads to death in almost $100 \%$ of cases. Emphysematous carbuncle is a serious problem of livestock industry, not only due to economic damage caused by the disease and death of animals, but also due to the emergence of persistent adverse situation in the area contaminated with pathogen spores and losses from the imposition of quarantine measures and restrictions. Cattle and small ruminants up to three years of age are mostly prone to get ill.
\end{abstract}

\section{KEY WORDS}

Etiology, pathogenesis, clinical signs, pathological changes, diagnostics, treatment, specific prevention of clostridiosis.

The pathogen of emphysematous carbuncle, Clostridium chauvoei, was isolated in 1876 by Bollinger and Feather, and then studied and described by Arloin and Thomas in 1881-1884, who named it after the French scientist J.B.A. Chauveau $[5,16,25]$.

Clostridia of this species are polymorphic spore-containing gram-positive mobile rods with rounded ends, $0.4 \times 0.6-2 \times 8$ microns in size, located singly or in short chains and they belong to strict anaerobes. The spores are located centrally, subterminally, or lie freely, the spores are oval in shape. In young, actively growing cultures, as well as in pathological material, the cells of the microorganism are stained gram-positive, during further cultivation they become gram-negative, and due to the forming spores, they acquire spindle or lemonshaped forms. Spores for a long time stay in the soil, posing a danger of infection of susceptible animals $[7,9,11,16,34]$.

On liquid nutrient media, they grow intensively with weak gas formation. They ferment glucose, fructose, galactose, maltose, sucrose and lactose with the formation of acid and gas, they liquefy gelatin, and slowly curdle milk. In brain medium they do not give blackening. They produce a small amount of indole. Turbidity during growth in Kitt-Tarozzi medium occurs after 16-20 hours, but after 30-48 hours the growth stops and a gradual clarification of the medium begins accompanied by appearance of the sediment, which, if vigorously shaken, breaks up into a uniform suspension.

On blood sugar agar it forms round colonies in the form of a nacre button or in the form of a grape leaf with a zone of hemolysis. 


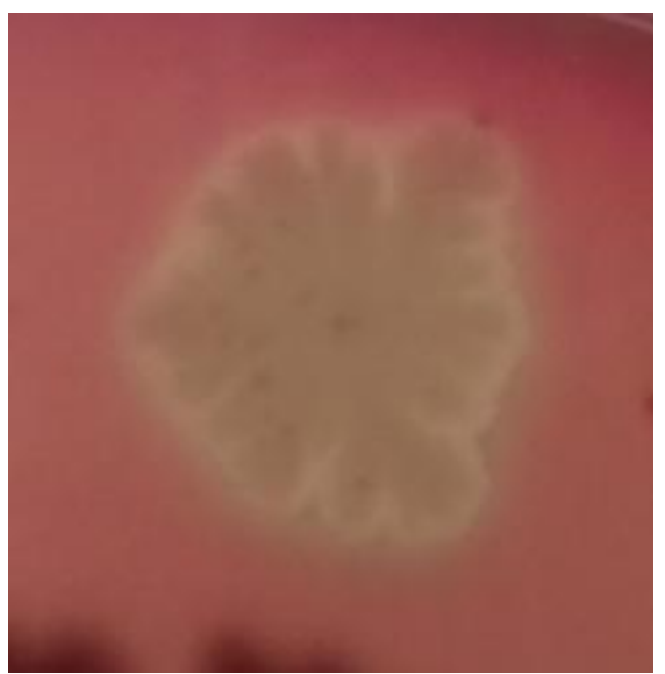

Figure 1 - Colony $\mathrm{Cl}$. chauvoei in the form of a grape leaf with a distinct zone of b-hemolysis

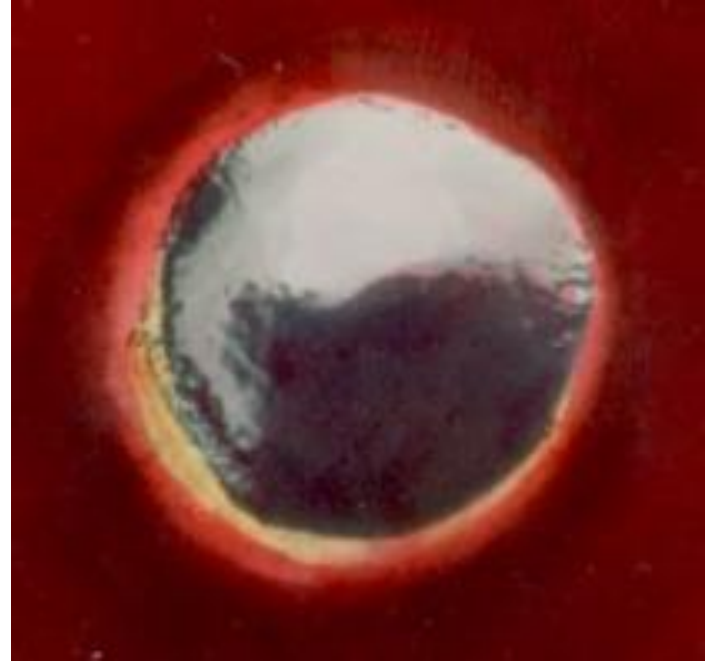

Figure 2 - Colony $\mathrm{Cl}$. chauvoei in the form of a nacre button with a distinct zone of b-hemolysis

Pathogenesis. Despite the lack of pronounced toxigenicity, Clostridium chauvoei has a significant number of virulence factors, such as the CctA toxin with hemolytic and cytotoxic activity, cholesterol-dependent cytolysins involved in the pathogenesis of gangrenous lesions, hyaluronidase, DNase, neuraminidase. In addition, it is believed that Cl. chauvoei flagella contribute significantly to the infectious process, providing the spread of the pathogen through the tissues of the body $[1,13,16,24,28]$.

Infection of animals often occurs through damaged membranes of the digestive tract, where bacteria get with feed contaminated by soil, less often open wound surfaces become the gate for infection. The transfer of the pathogen by horseflies is also considered proven $[16,26]$

Having overcome the mucosal barrier, Cl. Chauvoei is carried by blood to organs and tissues. It is localized in muscle tissue, where it begins to multiply, releasing gas, toxins and aggressins. Under their influence, there occur dystonia of the blood vessels, mucoid swelling of myocytes, an increase in the permeability of the capillary walls and the development of edema in the intermuscular tissue. Exotoxins and spores spread with edematous fluid and exudate to other organs and tissues. Increased intoxication of the body leads to the appearance of degenerative processes in the myocardium, lungs, liver, kidneys and other organs. The cause of animals' death is the paralysis of myocardium [10,16,23,27].

Clinical signs. The incubation period of emphysematous carbuncle lasts from 2 to 3 , less often up to 5 days. The disease is acute with a slight rise of temperature to $40.5-41^{\circ} \mathrm{C}$, but more often the body temperature of a sick animal remains within normal limits. In the most severe cases, animals are found dead without clinical signs. With natural infection, the first clinical signs of the disease are obvious lameness of one of the limbs and impaired coordination of movements. Within 36 - 48 hours in various parts of the body (more often in the muscles of the gluteal and femoral region, muscles of the neck and submandibular space) painful, dense, hot to the touch swellings are formed, when pressed sounds of crepitus are heard. The lesions are rapidly increasing in size. As the pathological process develops, the edemas become cold and painless. The disease is accompanied by lameness, oppression, hypotension of reticulum, rumen and omasum; hyperthermia, refusal of feed, stop of rumination, shortness of breath and tachycardia are also noted. In rare cases, when the tongue is involved, local swelling can cause the organ to bulge outside the mouth [16].

Animal dies within $24-72$ hours in almost $100 \%$ of cases.

Sometimes the disease proceeds subacutely and chronically, and in this case the disease manifests itself in the loss of appetite, mild oppression, colic, and small painful edemas of muscle tissue. Such cases, as a rule, end in recovery with the subsequent formation of persistent lifelong immunity. 
Pathological changes. The characteristic pathological signs of emphysematous carbuncle are severe swelling of the corpse due to intense gas formation in the reticulum, rumen, omasum and affected muscles; discharge of frothy bloody fluid from orifices. Specific lesions can be found in any muscle, including the heart, but they tend to appear in the most powerful muscles in the hips, croup, lower back, and shoulder. Often, the development of emphysematous carbuncle is preceded by injuries, including closed ones. Muscle tissue and intermuscular layers adjacent to necrotic lesions are infiltrated with serous exudate with gas bubbles; when cut at the region of the lesion, a bloody frothy liquid is released, which smells of rancid oil. The skin of the affected area is tense, thinned, blue-grey, sometimes necrotic. Wool comes off easily. Regional lymph nodes are in a state of serous-hemorrhagic lymphadenitis.

One of the most characteristic pathological changes provoked by emphysematous carbuncle is an overflowing gallbladder, enlarged 5-10 times compared with the norm $[16,24,29,32]$.

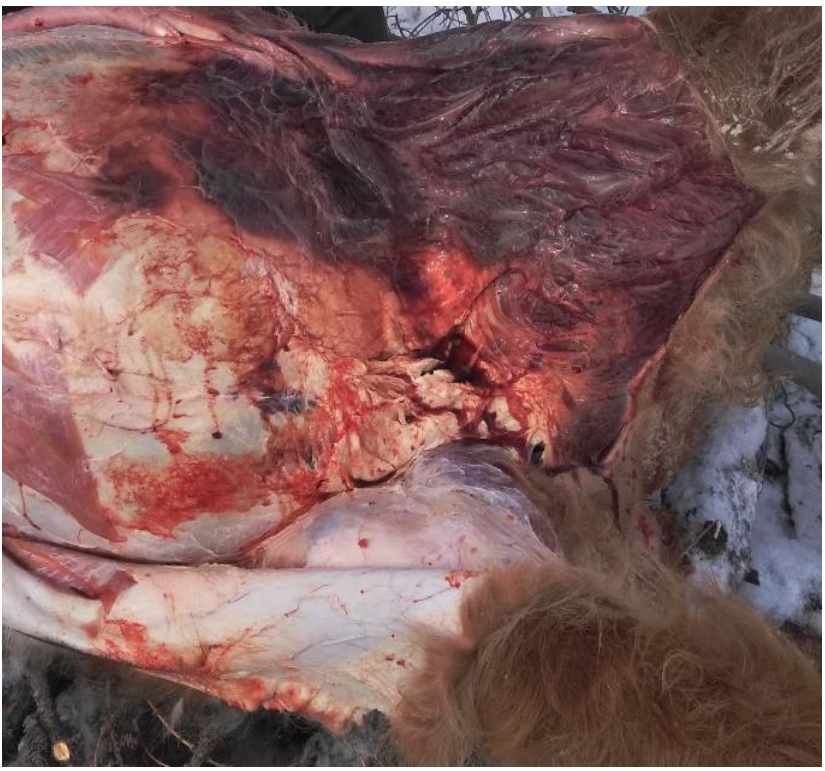

Figure 3 - Damage to the muscles of the left pelvic limb

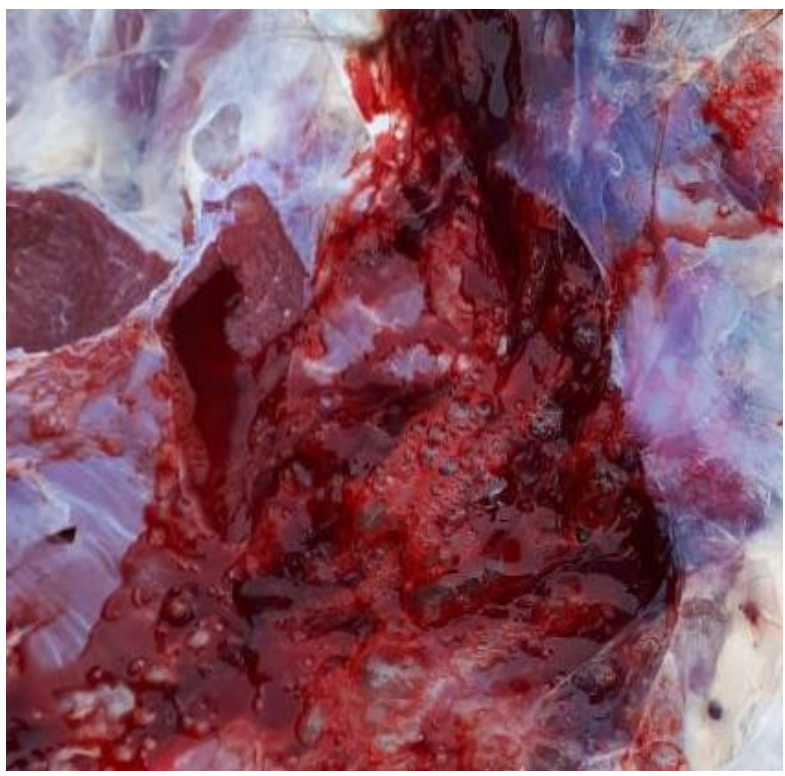

Figure 5 - Release of gas bubbles

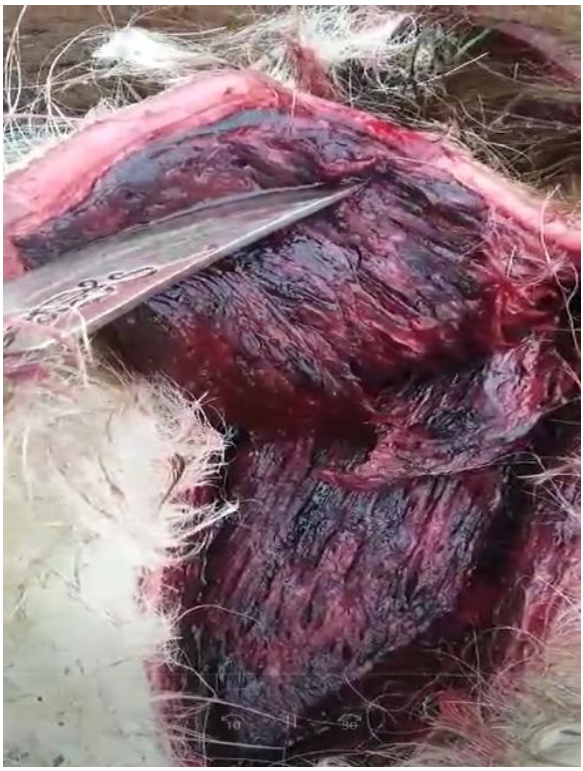

Figure 4 - Dark red, almost black coloration of muscle tissue in the lesion provoked by $\mathrm{Cl}$. chauvoei

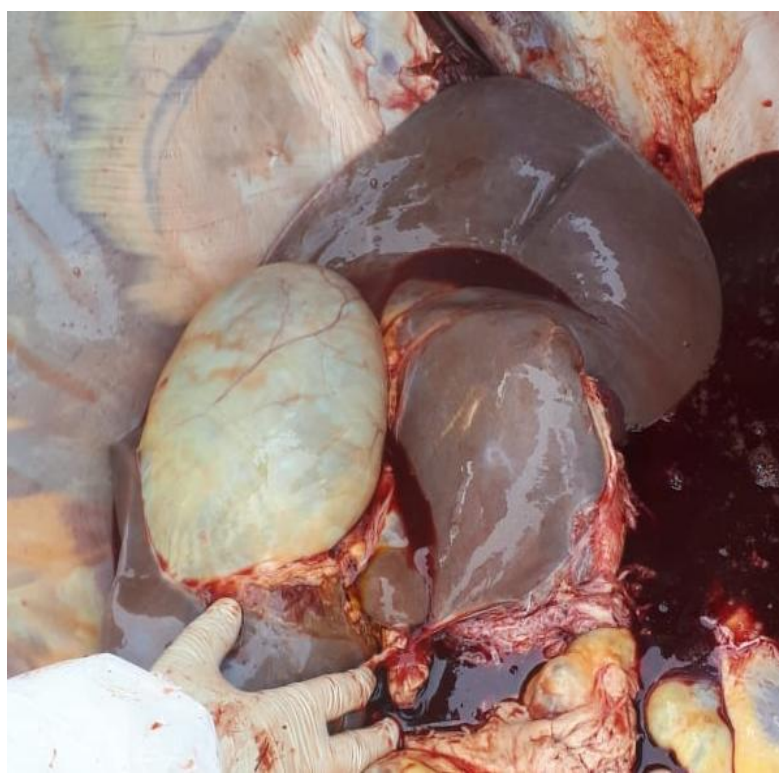

Figure 6 - Enlargement of the gallbladder due to emphysematous carbuncle 
In addition to skeletal muscles, significant lesions are found in the myocardium, while the pericardium is diffusely hemorrhagic, and can be covered with a thin layer of fibrin and contain a significant amount of fluid. Fibrinous pleurisy can be observed, in the areas adjacent to the heart; the lungs are always swollen.

It should be noted that if an emphysematous carbuncle is suspected, an autopsy is not performed, and the corpse is destroyed by incineration.

Diagnostics. The diagnosis of emphysematous carbuncle is made in a comprehensive manner, taking into account the epizootological data, the adversity of the area, clinical signs and characteristic pathological changes. Laboratory diagnostics consists of a bacteriological study of biological material carried out in accordance with GOST (State Standart) 26503-85 "Methods for laboratory diagnostics of clostridiosis".

Identification and differentiation is carried out by studying the cultural, morphological and biochemical characteristics of cultures [16]. At present, identification is greatly facilitated by the use of diagnostic test systems and PCR diagnostics [28].

The setting of a bioassay on guinea pigs that should die with characteristic clinical signs within 24-72 hours after the introduction of the material suspension is particularly important in confirming the diagnosis. Typical pathological signs are: serous-hemorrhagic effusion on the skin at the site of the lesion, while the skin is difficult to separate from the altered muscles. The chest and abdominal muscles are moist, dark red, almost black in colour. Despite the pronounced muscle lesions, the intestines remain unchanged.

If emphysematous carbuncle is suspected, whole fresh corpses are sent to the laboratory for diagnostic studies. For the study of large animals, they take from them: pieces of tissues and parenchymal organs (liver, kidneys, spleen), heart with tied vessels, muscles taken on the border of healthy and affected tissue, whole tubular bone.

Each sample of material is placed separately in sterile disposable containers or glassware, avoiding contamination of their external surface. Given the high sensitivity of Clostridia to oxygen, samples of the material are sent to the laboratory immediately after sampling in order to preserve the viability of pathogens and prevent overgrowth of the accompanying microflora.

The samples are transported and stored before the start of the study in a refrigerator at a temperature of $2-8^{\circ} \mathrm{C}$. If test-tubes with Amies transport medium, which ensures the viability of bacteria for 72 hours, are used for transporting samples to the laboratory it is allowed to store them at the temperature of $18-20^{\circ} \mathrm{C}$. If it is impossible to send the material to the laboratory immediately after sampling, it is frozen once and then transported and stored at the temperature of $-18^{\circ} \mathrm{C}$ and below.

For transportation of liquid material (tissue exudate, blood, urine) syringes can be used. After filling the syringe, the remaining air is removed from it while covering the needle with a sterile cotton swab. The needle is disinfected with $70 \%$ ethyl alcohol. The end of the needle is injected into a sterile rubber stopper for sealing and this way the syringe with the material is delivered to the laboratory. When a large amount of material ( $3 \mathrm{ml}$ or more) is collected, anaerobic bacteria can remain viable for 24 hours at room temperature $\left(18-20^{\circ} \mathrm{C}\right)$.

One of the criteria for the preliminary diagnosis of clostridiosis in animals is the detection of the pathogen in smears prepared from samples of pathological material. Blood, urine and exudate smears are made by applying a drop of the material to the glass, followed by uniform distribution of it over the glass surface. For microscopy of smears from organs and tissues, imprints are prepared. In order to do this, a piece of a tissue sample, taken, if possible, from the depth of the material under study, is cut in half and the fresh cut is pressed against the surface of the slide for several times. It is advisable to prepare imprints using the second part of the sample material, which is left after sowing imprints on agar media.

The imprints are dried, fixed in an alcohol mixture or over a burner flame and Gramstained. The presence of gram-positive rods and spores suggests the presence of clostridiosis pathogens.

The material transported for the study in test-tubes with transport media is not used for the preparation of imprints due to the high risk of contamination with foreign microflora, or the imprints are prepared after sowing the material on nutrient media. 
Sowing material on nutrient media. To isolate Clostridium chauvoei from material, samples of liquid and solid material are sown onto nutrient media. For sowing, freshly prepared liquid nutrient media are used, or they are activated before sowing by heating testtubes and vials in a boiling water bath for 15-20 minutes to remove the oxygen dissolved in it, and then quickly cooled in cold water. Dense material (pieces of organs and tissues) is sown onto the media by the method of imprint smear, or suspensions are preliminarily prepared.

Cultivation of sowings is carried out in an anaerostat with the temperature of $37^{\circ} \mathrm{C}$ for 24-48 hours. To create anaerobic conditions, special gas generating packages are used, or air is pumped out using a vacuum pump, given that a certain level of rarefaction is required for the cultivation of various species.

Biological isolation method. Simultaneously with the sowing, part of the prepared suspension of pathological material is used to infect susceptible animals, usually guinea pigs. Two guinea pigs are infected subcutaneously in the abdominal muscles or intramuscularly in a volume of 0.5 and $1.0 \mathrm{~cm} 3$. It is advisable to infect one guinea pig with a material which is a mixture of the suspension and an equal amount of $10 \%$ calcium chloride solution in a dose, since some types of Clostridia cannot cause disease and death of animals without the presence of inflammation in the body.

Observation of the animals continues for 72-96 hours. In cases of death of animals, sowing from the blood of the heart, as well as the liver is carried out on meat-peptone liver broth and blood agar by the sowing-imprint method in order to isolate a pure culture of the pathogen.

The method allows to clear pathological material from extraneous non-pathogenic microflora by passing through a guinea pig.

Additionally, imprints from the sites of sowing and the diaphragmatic surface of the liver are prepared, which makes it possible to differentiate certain types of clostridia. For example, in imprints from the diaphragmatic surface of the liver $\mathrm{Cl}$. chauvoei is found as single or paired cells, and $\mathrm{Cl}$. septicum in the form of chains and threads.

In the presence of a pure culture of the microorganism, which is confirmed by the detection of homogeneous cells in imprints and typical colonies on agar, a study of its biochemical properties is carried out to identify the type and kind of pathogen. Cultures of Clostridia of 24-48 h growth from Kitt-Tarozzi medium or a suspension of cells washed off the agar are sown into test-tubes with gelatin, pepted meat broth, litmus milk, a mix of semi-liquid nutrient media with the addition of carbohydrates or polyols. Then they are cultured in an anaerostat. The study results are assessed daily for 10 days.

Treatment. Treatment for emphysematous carbuncle, like any other anaerobic infection, should be primarily considered from an economic point of view. Treatment is often inadvisable due to extensive tissue damage and the subsequent loss of animal productivity even if the recovery of the animal is achieved. In addition to this, a sick animal releases a huge amount of the pathogen into the environment, which poses a potential danger of infecting other animals and humans. Therefore, in many cases, it is advisable to slaughter the animal with the subsequent disposal of the corpse and complete disinfection of the premises.

With a high value of the animal, in cases when the disease is just beginning and there is no pronounced tissue damage, treatment can be started. When carrying out therapeutic measures, the biological characteristics of anaerobes, pathogenesis and the form of disease manifestation are taken into account. In this case, unfavorable conditions for pathogens are created, neuro-dystrophic and gangrenous processes are prevented and the body defence system is stimulated.

Surgical treatment is of great importance. Animals with signs of anaerobic infection are isolated, all treatment procedures are carried out in a separate room, not connected with the hospital, dressing room or other rooms where other animals are kept or treated. Before the operation, anaesthesia is performed using novocaine blockades. While the dissection of the affected areas the dead tissue is excised as much as possible and the formation of pockets is not allowed. Tissues in the zone of gangrenous lesions are dissected with one or more wide incisions within the edema, up to the border of bleeding signs. Before, during and 
immediately after excision the wound is cleaned for a long time (up to 20 minutes) with hot ( $40^{\circ} \mathrm{C}$ ) hypertonic solutions of salts with hydrogen peroxide, potassium permanganate or chloramine. The cleaned wounds are loosely drained with the use of disinfecting bactericidal agents or generously powdered with antibiotics.

Rubber shoes, gloves, surgical underwear and instruments are thoroughly washed and sterilized. The equipment and premises where animals are treated and kept are disinfected. The dressing material and ropes contaminated with exudate are burned.

Oxygen therapy also has a pronounced therapeutic effect, in which oxygen from an oxygen pillow is pumped through a needle into the zone of the anaerobic infection and border healthy tissues.

It is clear that the treatment of anaerobic infection is impossible without the use of various antibiotics. To stop the causes of the disease and as therapeutic measures, it is advisable to use complex antimicrobial drugs in increased doses, while they are administered both for systemic action, and abundantly injected into tissues around the lesion.

Non-specific treatment manifests itself in the use of means and methods that alleviate the course of the disease. For anesthesia, novocaine-antibiotic blockade is used, intoxication is alleviated by removing various solutions, for example, $40 \%$ hexamethylenetetramine and caffeine, $10 \%$ calcium chloride solution with $20 \%$ glucose solution, $10 \%$ rheopolyglucin, etc.

Specific medicinal sera for the treatment and prevention of emphysematous carbuncle are not produced.

Specific prevention of clostridiosis. Due to the wide spread of the pathogen in the environment, the acute or hyperacute course of the disease, the severity of the damage to the tissues of the body, the treatment of animals is almost $100 \%$ ineffective. The main way to prevent emphysematous carbuncle, like other clostridiosis, is specific prevention measures.

The effectiveness of vaccines depends on many factors, such as the quantity and quality of the antigen, adjuvant, immunostimulants, technology of the medicine preparation, and the scheme of its application. The immune status of the animal's body also plays an important role.

Vaccination of animals against emphysematous carbuncle was first proposed by the French researchers Carnevin, Arloin and Thomas in 1882. As a vaccine material, they used a powder from dried and grated muscles of a sick animal, diluted with sterile water 1:2, which was injected twice with an interval of 10-12 days. To weaken the virulence, the material was heated for 7 hours at $95-100^{\circ} \mathrm{C}$. These experiments, however, did not give the expected effect, since when heated, the antigens of the pathogen, which have immunogenic properties, were destroyed. Later, many researchers reported the creation of both mono and associated drugs against clostridiosis in cattle and sheep, while the presence of the $\mathrm{Cl}$. chauvoei component in vaccines was unchanged. Starting from the 1930s, vaccines against emphysematous carbuncle, made from cultures inactivated with formalin, were almost simultaneously manufactured and began to be actively used in different countries.

In the USSR, fighting emphysematous carbuncle was a critical issue, so the vaccine against it was one of the first drugs created in the country. The author of the drug was S.N. Muromtsev, who proposed it in 1931. The production and the usage of it allowed getting away from the so-called "blacklegoids" previously used for immunization, which are boluses compressed from the powder of the muscles affected by emphysematous carbuncle, treated at $97^{\circ} \mathrm{C}$ for $5-6$ hours. This method of prevention was developed in Italy in 1908 by the researcher Kitt, and until 1918 "blacklegoids" were imported to Russia from abroad, and then they began to be produced at the Don, Dnepropetrovsk and Voronezh biofactories.

The vaccine proposed by Muromtsev was a culture of highly virulent $\mathrm{Cl}$. chauvoei grown in a nutrient medium and inactivated with formalin, which made it completely safe for animals and quite effective. In one immunizing dose the medicine contains at least 10 billion m.c. of strain $\mathrm{Cl}$. chauvoei $i R_{15}$, inactivated with formalin and adsorbed on aluminum hydroxide as an adjuvant. Another medicine is a live associated vaccine against anthrax and emphysematous carbuncle, made from attenuated strains of pathogens. 
Each of these drugs has its own advantages, the most important is a single injection, which allows to induce immunity for 6 months (from an inactivated vaccine) and 12 months (when using a live vaccine).

Currently, in the Russian Federation there are two more associated vaccines against clostridiosis, including emphysematous carbuncle. These are vaccines "Klostbovak-8" and "Antoks-9». There is also a number of foreign analogues. Vaccination against clostridiosis is applied to all highly productive livestock, both dairy and meat breeds. Given such a wide range of medication with a diverse composition of antigens, manufacturers use those of them that are most relevant in a particular area.

\section{REFERENCES}

1. Glotova, T.I. Pathogens and age-related predisposition of cattle to clostridiosis / T.I. Glotova, T.E. Terentjeva, A.G. Glotov // Siberian Bulletin of S.-kh. science. - 2017. - T.47. - No. 1. - p.90-96.

2. GOST 26503-85 Methods of laboratory diagnostics of clostridiosis // Moscow. - 1985. p.16.

3. Gryaznova, D.V. Development of complex drugs for the prevention and diagnosis of gas gangrene // Diss.... Cand. biologist. sciences. / D.V. Gryaznova - Perm. -1999. - p. 120.

4. Kagan, F.I. Production test of concentrate-vaccine against emphysematous carbuncle, malignant edema and pasteurellosis in cattle // F.I. Kagan, N.M. Nikiforova, A.I. Kolesova / Proceedings of SOCl - 1964. - p. 245-248.

5. Kagan, F.I. Specific prevention and therapy of anaerobic infections // Diss. ... Doctor vet. sciences. / Moscow. - 1964.- p.326.

6. Kagan, F.I. Associated immunization of animals // F.I. Kagan / Agricultural Biology. 1966. - Vol. 1. - p.491-496.

7. Kagan, F.I. Experience of simultaneous vaccination against emphysematous carbuncle and anthrax // F.I. Kagan, J.R. Kovalenko / Soviet Veterinary Medicine. - 193223-24. p.17-20.

8. Kagan, F.I. Production test of concentrate-vaccine against emphysematous carbuncle, malignant edema and pasteurellosis in cattle // F.I. Kagan, N.M. Nikiforova, A.I. Kolesova / Proceedings of SOCl. - No. 13. - 1966. - p.18-22.

9. Kapustin, A.V. Epizootology and prevention of clostridiosis in cattle // A.V. Kapustin, T.I. Aliper / One World - One Health. Materials int. vet. Congress. - 2017. - p. 106-108.

10. Kapustin, A.V. The effectiveness of the vaccine "Klostbovak-8" against clostridiosis of cattle caused by various species of Clostridium spp. // Kapustin A.V., Sklyarov O.D., Laishevtsev A.I. // Veterinary Medicine, Animal Science and Biotechnology. 2016.- No. 9.- p. 6-11.

11. Kapustin, AV, Determination of the concentration and frequency of administration of the C. chauvoei component in the development of associated vaccines. // Kapustin A.V., Glushenkova Yu.A. / Scientific basis for the production and quality assurance of biological products of the agro-industrial complex, materials of the scientific-practical conference dedicated to the 95th anniversary of the Armavir Biofactory - 2016. - p.229234.

12. Kapustin, A.V. Development of a vaccine against emphysematous carbuncle in cattle. / Kapustin A.V. // Russian Journal of Agricultural and Socio-Economic Sciences, 5 (53) 2016. - p. 97-102.

13. Kapustin, A.V. The problem of clostridia in dairy farming. / Marder V., Kapustin A.V., Shcherbakov P., Shilova E. // f. BIO. - No. 5 (188), 2016.- p. 34-38.

14. Kapustin, A.V. Species composition of cattle clostridia / Kapustin A.V., Motorygin A.V., Bukova N.K. // Bulletin of veterinary medicine, No. 1 (64) - 2013. - p. 71-73.

15. Kirillov, L.V. Standardization of vaccines, toxoids and antitoxic sera used for specific prophylaxis of clostridiosis // Diss. ... doct. veterinary sciences / L.V. Kirillov. - Moscow. 1984. - p.305. 
16. Kovalenko, Ya.R. Anaerobic infections of farm animals // Ya.R. Kovalenko / Moscow. 1954. - p. 338.

17. Mayansky, A.N. Microbiology for doctors // A.N. Mayansky / Clostridium. - Nizhny Novgorod. - 1999. - p. 139-152.

18. Panin, A.N. Basic requirements for production and control strains of microorganisms / A.N. Panin, N.T. Tatarintsev // Veterinary Medicine. - 1993. - No. 4. - p. 28-29.

19. Pervova, L.M. Development and testing of an associated vaccine against emphysematous carbuncle and leptospirosis in cattle // Diss. Cand. veterinary sciences / L.M. Pervova. - Moscow. - 1988 - p. 135.

20. Pleskikh, S.A. Development and testing of a live associated vaccine against anthrax and emphysematous carbuncle in cattle // Diss. ... Cand. vet. sciences / S.A. Pleskikh Moscow - 1992. - p. 131.

21. Radionova, K.P. Clostridiosis of farm animals / K.P. Radionova, O.V. Karabanova // Veterinary of agricultural animals. - 2010. - No. 9. - p. 12-14.

22. Redkozubova, L.I. Control of clostridia - systematic vaccination / L.I. Redkozubova // Veterinary Medicine. - 2016. - t. No. 1. - p. 9-12.

23. Storozhev, L.I. Comparative study of live and killed vaccines against emphysematous carbuncle // Diss ... Cand. Veterinary Sciences / L.I. Storozhev - 1977 - p. 168.

24. Terentjeva, T.E. Species spectrum of bacteria of the genus Clostridium isolated from cattle on dairy complexes / T.E. Terentjeva, Glotov A.G., Glotova T.I., Koteneva S.V. // Russian vet. magazine. - Issue. 1.- 2016. - p.5-9.

25. Urguev, K.R. Clostridiosis of animals // K.R. Urguev / Rosselkhozizdat. - Moscow. - 1987 - p. 183.

26. Shatiko, P.D. The role of horseflies as carriers of emphysematous carbuncle in cattle. / P.D. Shatiko. - well. Veterinary Medicine - No. 7 - 1952 p. 76-79.

27. Frey, J. Cytotoxin CctA, a major virulence factor of Clostridium chauvoei conferring protective immunity against myonecrosis // J. Frey / Vaccine. - 2012. - 30. - p.55005505.

28. Kuhnert, P. Identification of Clostridium chauvoei in cultures and clinical material from blackleg using PCR // P. Kuhnert, M. Krampe, S.E. Capaul, J. Frey, J. Nicolet / Vet. Microbiol. - 1997. - 57:291-298.

29. Giraudo Conesa, L.C. Detection of Clostridium chauvoei in formalin-fixed, paraffinembedded tissues of sheep by the peroxidase-antiperoxidase (PAP) technique // L.C. Giraudo Conesa, S.A. Vannelli F.A. Uzal / Vet. Res. Commun. - 1995. -19. - p.451- 456.

30. Prescott, J.F. Clostridial infections // J.F. Prescott, D.S. McVey / Veterinary Microbiology, 3rd edition. - p.245.

31. Songer, J.G. Molecular and immunological methods for the diagnosis of clostridial diseases. In: The clostridia: molecular biology and pathogenesis // J.G. Songer, J.L. Rood, B.A. McClane R.W. Titball / Academic Press, Inc. USA. - 1997. - p.491-503.

32. Songer, JG. Clostridial diseases of small ruminants // JG. Songer / Vet Res. - 1998. - 29: p. 219-32.

33. Titball, R. Protein toxins of the genus Clostridium and vaccination. Scientific booklet of the CA QLK2-CT2001- 01267 // R. Titball, J. Mainil, C. Duchesnes, M. Popoff / Presses Fac. Méd. Vét. ULg, Liège, Belgium. - 2003 - p.89-99.

34. Wiegel, J. Family I. Clostridiaceae. In: De Vos, P. et al. (eds) Bergey's Manual of Systematic Bacteriology // J. Wiegel / Springer Science, New York. - p.736. 